\title{
Arthrofibrosis in Post-Anterior Cruciate Ligament Reconstruction: Remain a Challenging Dilemma
}

\author{
Hussin $\mathrm{AR}^{1}$, Miswan $\mathrm{MF}^{2}$, Tahir $\mathrm{SH}^{3}$ \\ ${ }^{1,3}$ Department of Orthopaedic and Traumatology, Hospital Kuala Lumpur, Jalan Pahang, 50586,Kuala Lumpur, Malaysia \\ ${ }^{2}$ Department of Orthopaedic Surgery, Faculty of Medicine, Universiti Teknologi MARA, \\ Jalan Hospital, 47000, Sungai Buloh, Selangor, Malaysia
}

\begin{abstract}
Pain and loss of motion of the knee after anterior cruciate ligament reconstruction remain a challenging dilemma. Arthrofibrosis is one of the causes for this problem. Our patient is a young motivated and compliant athlete who underwent anterior cruciate ligament reconstruction who experienced gradual loss of full knee extension within 3 months post operation. Examination showed firm painful swelling over the anteromedial aspect of the knee. Ultrasound suggested of synovial membrane thickening with irregularity pattern over the localized swelling and tenderness area. Arthroscopic adhesiolysis was performed and finally patient regained full extension and flexion. As conclusion, prevention is still the best form of treatment or otherwise early recognition is needed for early intervention.
\end{abstract}

Keywords: arthrosfibrosis, loss of knee extension, membrane thickening, adhesiolysis

\section{Introduction}

Pain and loss of motion of the knee after anterior cruciate ligament reconstruction remain a challenging dilemma. There are many causes for this problem that one of it is due to arthrofibrosis. Arthrofibrosis is a condition where an excessive scar tissue was formed in the joint that give restriction to the knee motion as well as pain. Even though there has been many study had been done as a result of its frequency of occurrences, prevention still the best form of treatment or otherwise early recognition is needed for early intervention [1].

\section{Case Report}

23-year-old female karate athlete sustained isolated complete torn anterior cruciate ligament of her left knee. Anatomical single bundle reconstruction using quadruple loop of hamstring sized $8 \mathrm{~mm} \times 100 \mathrm{~mm}$ was done four weeks after the injury. Intraoperative was uneventful. Femoral tunnel was $8 \mathrm{~mm} \times 36 \mathrm{~mm}$ with button loop $15 \mathrm{~mm}$ and tibia tunnel size 8 with bio-screw $10 \mathrm{~mm} \times 25 \mathrm{~mm}$ to fix the graft. Upon discharged from ward on day five post operation noted patient was able to perform full active knee extension. She was very compliant to rehabilitation program and also performed the exercises at home. However on follow up in clinic, patient started to loss her active and gradually on subsequent visit later affected her passive full extension (10 degrees extension lag) at 3 months post operation. Regional examination showed diffused firm swelling over the anteromedial aspect of the knee just above the medial joint line that was very tender. Radiological $x$-ray found no significant findings. Hence ultrasound was performed suggested of synovial membrane thickening with irregularity pattern over the localized swelling and tenderness area.

\section{Results}

Before diagnostic arthroscopy procedure, manipulation under anaesthesia (MUA) was performed. Her knee was initially unable to be fully extended. The range of motion passively was from $10^{\circ}$ to $150^{\circ}$. Her flexion was almost equal compared to opposite knee. Then diagnostic arthroscopy was performed. The finding showed thick scar tissue located at the suprapatellar pouch (Figure 1) and mainly over the medial gutter (Figure 2). There was no synovial impingement, meniscus tear, loose body or cyclope lesion noted. Adhesiolysis was performed during the arthroscopic procedure which then range of knee motion was checked intraoperative which noted the knee could be passively full extended and flexed comparable to non affected knee.

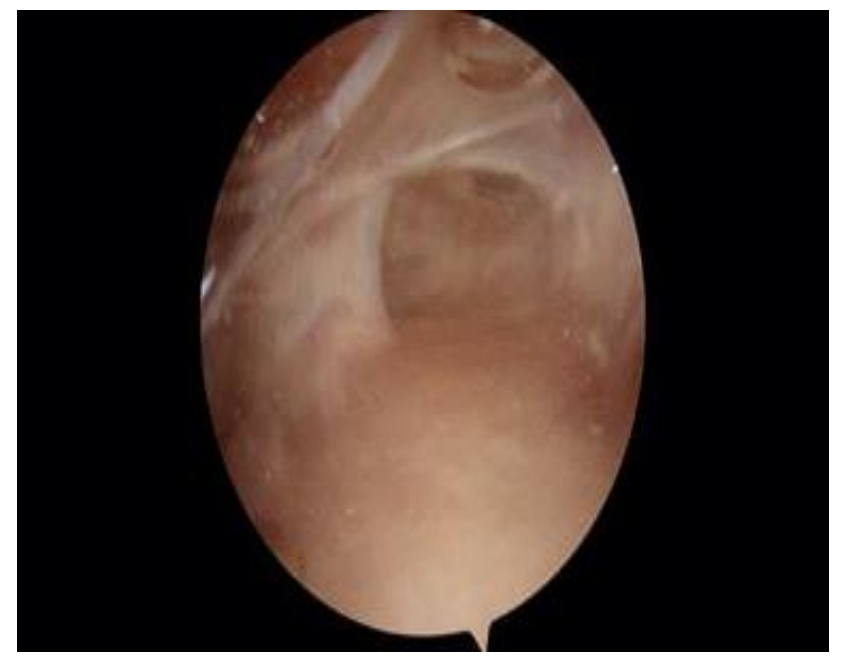

Figure 1: Fibrous tissue adhesion over suprapatella pouch. 


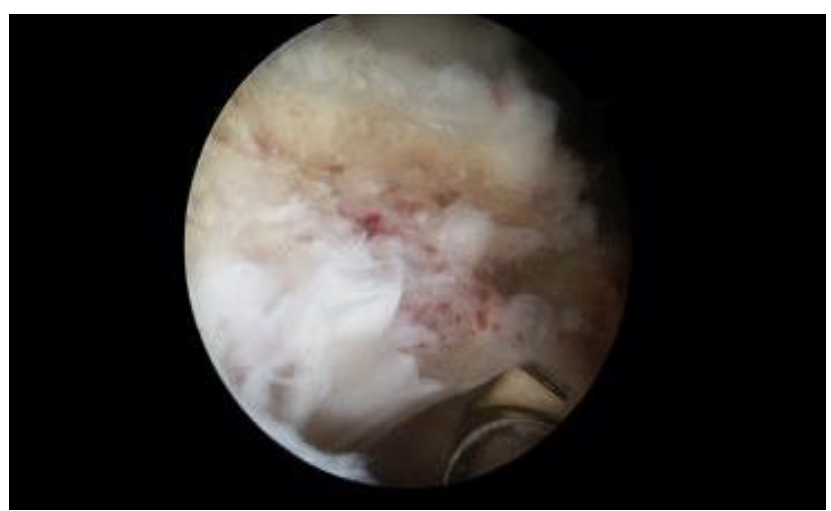

Figure 2: Adhesiolysis performed

\section{Discussion}

Arthrofibrosis can disrupt the normal knee kinematics and may lead to progressive degenerative changes in the knee [2]. Although the knee is stable by objective criteria, the patient is often dissatisfied and frequently seeks treatment because of persistent symptoms and impairment of knee function. There is a research being done to identify at-risk patient, looking into association between the development of arthrofibrosis and specific allergic groups of the human leukocyte antigen [3]. Despite taking these measures, the stiffness of the knee may still arise. In conclusion, early detection with early intervention is very important in determining the outcome of the treatment.

\section{References}

[1] Achalandabeso J, Albillos J. Stiffness of the knee - mixed arthroscopic and subcutaneous technique: results of 67 cases. Arthroscopy 9: 685-690, 1993.

[2] Paulos LE, Rosenberg TD, Drawbert J, et al. Infrapatella contracture syndrome. An unrecognized cause of knee stiffness with patella entrapment and patella infera. Am J Sports Med 15: 331-341, 1987.

[3] Skutek M. Elsner HA, Slateva K, et al. Screening for arthrofibrosis after anterior cruciate ligament reconstruction: analysis of association with human leukocyte antigen. Arthroscopy 2004; 20:469-473.

\section{Author Profile}

Abdul Razak Hussin received the M.D. and M.S. degrees in Orthopaedic from Universiti Kebangsaan Malaysia in 1998 and 2010, respectively. He now is doing fellowship in Sports injury and Arthroscopy Surgery (2015-2017). 